\title{
Clearly We've Only Just Begun: Developing Effective Prevention Programs for Intimate Partner Violence
}

\author{
Jennifer Langhinrichsen-Rohling • Deborah M. Capaldi
}

Published online: 1 July 2012

(C) Society for Prevention Research 2012

Articles included in this Special Section present evidence that supports an encouraging new vision for prevention of intimate partner violence (IPV) in dating relationships and also for interventions with many couples experiencing IPV. This paradigm shift involves focusing on the role of dyadic interactions in the production of violence, while highlighting the necessity of addressing risk factors for IPV perpetration by both members of the romantic dyad (even if only one partner is participating in the prevention program)-be the participant a man or a woman. This work is part of a growing body of evidence pointing in this direction (e.g., Archer 2000; Capaldi et al. 2007; Langhinrichsen-Rohling 2010). As articulated by Pepler (2012) and LanghinrichsenRohling and Turner (2012), preventing IPV requires an understanding of the characteristics that constitute a healthy romantic relationship. Specifically, as delineated by the Centers for Disease Control (2008), these characteristics include: (a) belief in nonviolent conflict resolution; (b) effective communication skills; (c) the ability to negotiate and adjust to stress; (d) belief in partner's right to autonomy;

(e) shared decision making; and (f) trust.

This dyadically focused vision of the development of IPV challenges some of the prevailing beliefs about gender and IPV; beliefs that form the basis of many existing yet relatively ineffective treatment interventions for IPV perpetrators (Babcock et al. 2004; Dutton 2012; Ehrensaft 2008; Whitaker et al. 2006). One such belief is that IPV is typically

J. Langhinrichsen-Rohling

University of South Alabama,

Mobile, AL, USA

D. M. Capaldi $(\bowtie)$

Oregon Social Learning Center,

Eugene, OR, USA

e-mail: deborahc@oslc.org a one-sided behavior rather than incorporating the finding that IPV is more often bidirectional. Others include the beliefs that IPV is almost universally a male-to-female event, that IPV only functions as an instrumental behavior rather than one that is also related to anger and impulsivity, and that all certified IPV interventions should be based on long-standing gendered paradigms about domestic violence. Further, as argued by Dutton (2012) and advocated by Teten Tharp (2012), a focus on prevention is likely to be more effective than criminal justice interventions and mandated batterer treatments, as these occur after violent conflict management and dominance strategies have solidified over time. Early prevention and intervention efforts may also be considerably less costly and have other ancillary benefits, including improving the overall stability of families and the well-being of children by enhancing couples' interaction skills.

Highlights of the research contained within this Special Section that support the necessity of a new vision for IPV prevention include evidence that (a) IPV is predominantly mutual rather than one sided (Chiodo et al. 2012; O'Leary and Slep 2012); (b) IPV is dyadic and interactive in nature such that the probability of violence changes based on the nature of one's current partner and relationship (O'Leary and Slep 2012; Shortt et al. 2012); thus, a focus on dyadic rather than only individual behavior is of key importance; (c) IPV is predictable for both boys and girls using psychological constructs and developmental trajectory methodologies (Chiodo et al. 2012; O’Leary and Slep 2012); (d) at-risk populations can respond favorably to prevention programs (Langhinrichsen-Rohling and Turner 2012); and (e) early prevention programs that have the potential to target broad risk factors and peer and family based patterns can have long-term positive effects on individual and dyadic functioning (Ehrensaft and Cohen 2012; Reyes et al. 2012). 
Teten Tharp (2012) furthers the discussion of the importance of IPV prevention by highlighting the Centers for Disease Control's new comprehensive dating violence prevention initiative: Dating Matters ${ }^{\mathrm{TM}}$ : Strategies to Promote Healthy Teen Relationships. This universal prevention program is being targeted at 11- to 14-year-old youth to build skills prior to their experience of relationship violence, and it is built on the premise that it is essential to directly address both individual and relationship risk factors (Teten Tharp 2012).

These current findings regarding dyadic influences lead to the exciting and important conclusion that, even with regard to IPV, women appear to be influential in their relationships with their male partners and thus more powerful and agentic in this regard than has been typically assumed. This is not akin to blaming women for their victimization. Instead, it appears that many women do not merely have a choice between accepting victimhood or leaving the relationship; they can also stay in some relationships and effect change. Further work is needed to determine which choice is most likely to be successful for which relationships containing IPV. This conclusion is in keeping with Pepler's (2012) assertion that IPV falls along a continuum that often includes playful aggression that is not coded as problematic by either partner. However, the continued occurrence and persistence of even playful aggression in adolescent romantic relationships may signal that there are deficits in that couples' ability to problem solve constructively.

Other evidence related to the importance of considering dyadic processes when making treatment plans includes findings within these current studies that suggest that IPV is responsive to the interactional processes that emerge between partners, such that continuity in one partner's IPV is dependent on whether the other partner is also aggressive (Shortt et al. 2012). By implication, if women reduced their levels of IPV within an ongoing mutually aggressive relationship, men would also be likely to reduce their IPV. A mutual reduction in the use of IPV, however, is most likely to occur in the context of both members of the couple acquiring better skills for self-regulation and conflict resolution (Pepler 2012). It is also more likely to occur in relationships in which both partners actively work to foster empathy for each other while minimizing power struggles and dominance motives (Pepler 2012)

Second, a very interesting finding emerged from the Langhinrichsen-Rohling and Turner (2012) study. Although their intervention only involved the teen mothers and addressed the women's relationship skills and overall relationship aggression issues (i.e., male partners were not included in the intervention), the level of their male partner's severe physical IPV, and thus the women's victimization, significantly decreased across the course of the intervention. Although this is a small study and the effect needs replication, these results were certainly supportive of the notion that violence in relationships can be impacted by changing the dyadic relationship behavior of either partner.

Findings regarding types of IPV behaviors on which to focus indicate that IPV prevention programs need to consider the occurrence of both physical and psychological violence. Including a focus on psychological violence is essential because it is both extraordinarily common in young people's romantic relationships and it may be one of the most robust predictors of IPV perpetration (O'Leary 1999). Electronic communication also now plays a significant role in the initiation, maintenance, and dissolution of romantic relationships (Draucker and Martsolf 2010), and dating web sites are involved in mate selection. Psychological abuse of a partner may occur electronically in addition to in person. Programs that acknowledge and target the full range of aggressive behaviors (psychological, physical, sexual, stalking, cyberaggression) are likely to be more successful than programs that consider each type of abuse independently, because many types of violence share common risk and protective factors and co-occur in relationships (e.g., O'Leary and Woodin 2009). Further research is needed on how to harness technology to facilitate access, compliance, and delivery of effective IPV interventions to youth.

Regarding risk factors for IPV, the articles by Chiodo et al. (2012) study and O'Leary and Slep (2012), along with other studies (Ehrensaft et al. 2003), provide evidence that developmental histories and long-standing risk factors for IPV (insecure attachment patterns; Langhinrichsen-Rohling and Turner 2012) show similarities for boys and girls, particularly in that higher levels of conduct problems and prior aggression are a risk factor for IPV perpetration for both genders. In the commentary by Pepler (2012), she argues the importance of utilizing a developmental-systemic perspective of IPV. This perspective yields a greater understanding of the links between early familial stress experiences, childhood bullying, substance abuse issues in early adolescence, use of aggression to achieve dominance or to minimize large gaps in advantage across a culture, and the development of IPV. Girls and boys following this type of violence-prone trajectory tend to be attracted to one another, a finding that increases the risk of bidirectional violence within those romantic relationships. This conclusion is supported by the research of Chiodo et al. (2012). They found that girls in dating relationships involving mutual violence showed the poorest overall adjustment (including higher levels of delinquency as well as the highest levels overall of other forms of aggression toward peers, distress, and suicidal thoughts) compared with girls who were only victims or perpetrators of IPV. The determination that there may be a cluster of risk behaviors in girls involved in more problematic levels of IPV, particularly mutual IPV, needs to be addressed in a 
more forthright fashion by those designing teen dating violence prevention programs as well as by those addressing adolescent mental health for girls more generally.

Special attention may be needed to understand the interactions among alcohol use, substance abuse, and IPV perpetration and victimization (Friend et al. 2011). Reyes et al. (2012) found that heavy alcohol use was associated with dating violence perpetration in adolescence and that this association increased as levels of family conflict and friend involvement in dating violence increased. They found no evidence of differences between boys and girls in these associations. However, a number of recent studies have indicated that there is a complex interplay among childhood experiences, alcohol use, and IPV for men versus women (Maas et al. 2010; Rapoza and Baker 2008; Schnurr et al. 2010). The association of use of substances other than alcohol and IPV is not well understood, and there are few studies to date that can tease apart how much IPV may be because of alcohol use versus co-occurring antisocial behavior or other forms of substance use (Feingold et al. 2008).

There are also some potential risk factors for IPV that have been understudied, particularly the role that peers can play in the romantic relationships of youth. Accumulating evidence indicates that peers may have important influences on the production of and reaction to violence (Connolly and Friedlander 2009) and that bullying of peers in childhood may be a crucial risk factor for later IPV (Pepler 2012). Peer relationships may also interact with other risk factors for IPV. For example, Reyes et al. (2012) demonstrate that the association between heavy drinking and IPV is stronger for teens that have been exposed to higher rather than lower levels of peer violence. Violence in multiple types of relationships is another possible risk factor that warrants further attention, as youth who perpetrate aggression in both peer and dating contexts may be at greater risk for ongoing adult IPV (Ellis et al. 2008). The role of violence with siblings is another risk factor that has received relatively little attention in relation to IPV.

Findings regarding risk factors for IPV, such as delinquency and experiences of childhood distress, indicate that family based interventions may serve both to prevent IPV and to mitigate other related problems. Childhood maltreatment, family conflict, and poor parenting practices have been shown to have a number of deleterious outcomes over time (Ehrensaft and Cohen 2012; Wolfe et al. 2009), and parental relationships may function as either risk or protective factors for IPV (e.g., Nicodemus et al. 2009). Family based interventions such as the Strengthening Families Program (Kumpfer 2008), which includes a broad focus on effective parenting skills and parent-child relationships, may also help prevent IPV via the impact of generalizing relationships skills across contexts. Moreover, this type of family based intervention has the possibility of interrupting the intergenerational transmission of IPV, delinquency, and other types of externalizing behaviors (Ehrensaft and Cohen 2012; Maas et al. 2010). Research is needed to test these assumptions.

Overall, on a practical note, the findings contained within the current papers suggest that programs that are based on a one-sided model of IPV are likely to be ineffective for a number of reasons including, but not limited to, program participants' own opposition to exclusion of dyadic interaction skills from the program. If the descriptions and interpretations of IPV presented within any IPV program do not fit well with the participants' own experiences of IPV, unnecessary participant resistance may be generated. For example, youth are likely to know that the experience of mutual IPV is a common pattern (O’Leary and Slep 2012) and that not all of girls' IPV is motivated by self-defense; therefore, acknowledgement of the dyadic nature of much IPV is likely to engender participant buy-in to the prevention program while enhancing therapist creditability.

Regarding the timing of delivering IPV initiatives, it is possible that prevention programs will fare best if they are offered at times of transitions (e.g., as youth move into middle school or high school, at times of relationship instability, or during the transition to parenthood). These may be times when individuals are more receptive and motivated for change (Langhinrichsen-Rohling and Turner 2012; Shortt et al. 2012). However, given that the prevalence of IPV diminishes as people age out of adolescence, offering universal relationship-enhancing prevention programs to middle school-aged youth also seems warranted (Pepler 2012; Teten Tharp 2012).

Taken as a whole, these papers demonstrate that the IPV field is no longer in its infancy; the next generation of IPV prevention efforts is being launched (LanghinrichsenRohling and Turner 2012; Taylor et al. 2010; Teten Tharp 2012). These efforts can be conceptualized as having a developmental-systemic (Pepler 2012) or dynamic developmental systems (Capaldi and Langhinrichsen-Rohling 2012) perspective on IPV. The importance of retaining a focus on gender roles in these new efforts remains controversial as research indicates that the majority of risk factors for IPV apply to both male and female perpetrators (Medeiros and Straus 2006). However, gender identity continues to be a central aspect of an individual's sense of self and ability to interact successfully with a relationship partner (White 2009), and some investigators continue to assert that dating violence is a deeply gendered phenomenon (Zurbriggen 2009). Construction of a dynamic developmental systemsbased prevention program that maintains a degree of gender sensitivity is likely to be ideal.

Well-designed longitudinal studies, such as those included in this Special Section, have shed light on the importance 
of partner selection and fit, peer and community influences, and the general decline in IPV perpetration as people age (e.g., Chiodo et al. 2012; O’Leary and Slep 2012; Shortt et al. 2012), as well as the need to consider both individual and dyadic risk factors (Reyes et al. 2012). To be most effective, dating violence prevention programs will also need to be tailored to particular at-risk groups and types of communities (Jain et al. 2010). Even universal programs will need to be culturally sensitive in their application (Foshee et al. 2009). Prevention programs must be flexible to fit with societal changes relative to gender roles, the acceptability of men and women's violence in relationships for some individuals and groups, family structure and dynamics, minority status, substance use and accessibility, levels of poverty and the prevalence of significant gaps in advantage, and communication modes including electronic communications, as all of these factors relate to the perpetration of IPV and/or to the design and delivery of prevention programs.

It is unfortunate that "clearly we have only just begun" to design prevention programs that are based on empirical evidence from well-designed studies regarding the etiology and course of IPV. Too many resources of time, effort, and money have gone into previous IPV prevention and treatment programs that were not grounded in the research evidence and are therefore more likely to fail. The papers and commentaries in this Special Section provide evidence for and articulate a new prevention vision that is predicated on a well-supported dynamic developmental-systemic model. This vision warrants serious consideration by researchers, helping professionals, and advocates.

\section{References}

Archer, J. (2000). Sex differences in aggression between heterosexual partners: A meta-analytic review. Psychological Bulletin, 126, 651-680.

Babcock, J. C., Green, C. E., \& Robie, C. (2004). Does batterers' treatment work?: A meta-analytic review of domestic violence treatment outcome research. Clinical Psychology Review, 23, 1023-1053.

Capaldi, D. M., Kim, H. K., \& Shortt, J. W. (2007). Observed initiation and reciprocity of physical aggression in young, at-risk couples. Journal of Family Violence, 22, 101-111.

Capaldi, D. M., \& Langhinrichsen-Rohling, J. (2012). Informing intimate partner violence prevention efforts: Dyadic, developmental, and contextual considerations. Prevention Science.

Centers for Disease Control (2008) Strategic direction for intimate partner violence prevention: Promoting respectful, nonviolent intimate partner relationships through individual, community, and societal change. Retrieved from www.cdc.gov/injury

Chiodo, D., Crooks, C. V., Wolfe, D. A., McIssac, C., Hughes, R., \& Jaffe, P. G. (2012). Longitudinal prediction and concurrent functioning of adolescent girls demonstrating various profiles of dating violence and victimization. Prevention Science.
Connolly, J., \& Friedlander, L. (2009). Peer group influences on adolescent dating aggression. The Prevention Researcher, 16, 8-11.

Draucker, C. B., \& Martsolf, D. S. (2010). The role of electronic communication technology in adolescent dating violence. Journal of Child and Adolescent Psychiatric Nursing, 23, 133-142.

Dutton, D. G. (2012). The prevention of intimate partner violence. Prevention Science.

Ehrensaft, M. K. (2008). Intimate partner violence: Persistence of myths and implications for intervention. Children and Youth Services Review, 30, 276-286.

Ehrensaft, M. K., \& Cohen, P. (2012). Contribution of family violence to the intergenerational transmission of externalizing behavior. Prevention Science.

Ehrensaft, M. K., Cohen, P., Brown, J., Smailes, E., Chen, H., \& Johnson, J. (2003). Intergenerational transmission of partner violence: A 20-year prospective study. Journal of Consulting and Clinical Psychology, 71, 741-753.

Ellis, W. E., Crooks, C. V., \& Wolfe, D. A. (2008). Relational aggression in peer and dating relationships: Links to psychological and behavioral adjustment. Social Development, 18, 253-269.

Feingold, A., Kerr, D. C. R., \& Capaldi, D. M. (2008). Associations of substance use problems with intimate partner violence for at-risk men in long-term relationships. Journal of Family Psychology, 22, 429-438.

Foshee, V. A., Benefield, T., Suchindran, C., Ennett, S. T., Bauman, K. E., Karriker-Jaffe, K. J., Reyes, H. L. M., \& Mathias, J. (2009). The development of four types of adolescent dating abuse and selected demographic correlates. Journal of Research on Adolescence, 19, 380-400.

Friend, J., Langhinrichsen-Rohling, J., \& Eichold, B. (2011). Sameday substance use in men and women prosecuted for felony domestic violence. Criminal Justice and Behavior, 38, 619-633.

Jain, S., Buka, S. L., Subramanian, S. V., \& Molnar, B. E. (2010). Neighborhood predictors of dating violence victimization and perpetration in young adulthood: A multilevel study. American Journal of Public Health, 100, 1737-1744.

Kumpfer, K. L. (2008). Why are there no effective child abuse prevention parenting interventions? Substance Use \& Misuse, 43, $1262-1265$.

Langhinrichsen-Rohling, J. (2010). Controversies involving gender and intimate partner violence in the United States. Sex Roles, 62, 179-193.

Langhinrichsen-Rohling, J., \& Turner, L. A. (2012). The efficacy of an intimate partner violence prevention program with high-risk adolescent girls: A preliminary test. Prevention Science.

Maas, C. D., Fleming, C. B., Herrenkohl, T. I., \& Catalano, R. F. (2010). Childhood predictors of teen dating violence perpetration. Violence and Victims, 25, 131-149.

Medeiros, R. A., \& Straus, M. A. (2006). A review of research on gender differences in risk factors for physical violence between partners in marital and dating relationships. Durham, NH: Family Research Laboratory, University of New Hampshire. Retrieved from http://pubpages.unh.edu/ mas2

Nicodemus, P., Davenport, P. A., \& McCutcheon, L. E. (2009). The effects of maternal relationships on physical and psychological dating violence. North American Journal of Psychology, 11, 455-462.

O'Leary, K. D. (1999). Developmental and affective issues in assessing and treating partner aggression. Clinical Psychology: Science and Practice, 6, 400-414.

O’Leary, K. D., \& Slep, A. M. S. (2012). Prevention of partner violence by focusing on behaviors of both young males and females. Prevention Science.

O’Leary, K. D., \& Woodin, E. M. (Eds.). (2009). Psychological and physical aggression in couples: Causes and interventions. Washington, DC: American Psychological Association. 
Pepler, D. (2012). The development of dating violence: What doesn't develop, what does develop, how does it develop, and what can we do about it? Prevention Science.

Rapoza, K. A., \& Baker, A. T. (2008). Attachment styles, alcohol, and childhood experiences of abuse: An analysis of physical violence in dating couples. Violence and Victims, 23, 52-65.

Reyes, H. L. M., Foshee, V. A., Bauer, D. J., \& Ennett, S. T. (2012). Heavy alcohol use and dating violence perpetration during adolescence: Family, peer and neighborhood violence as moderators. Prevention Science.

Schnurr, M. P., Lohman, B. J., \& Kaura, S. A. (2010). Variation in late adolescents' reports of dating violence perpetration: A dyadic analysis. Violence and Victims, 25, 84-100.

Shortt, J. W., Capaldi, D. M., Kim, H. K., Kerr, D. C. R., Owen, L. D., \& Feingold, A. (2012). Stability of intimate partner violence by men across 12 years in young adulthood: Effects of relationship transitions. Prevention Science.

Taylor, B. G., Stein, N., \& Burden, F. F. (2010). Exploring gender differences in dating violence/harassment prevention programming in middle schools: Results from a randomized experiment. Journal of Experimental Criminology, 6, 419-445.

Teten Tharp, A. (2012). Dating matters ${ }^{\mathrm{TM}}$ : The next generation of teen dating violence prevention. Prevention Science.

Whitaker, D. J., Morrison, S., Lindquist, C., Hawkins, S. R., O’Neil, J. A., Nesius, A. M., Mathew, A., \& Reese, L. (2006). A critical review of interventions for the primary prevention of perpetration of partner violence. Aggression and Violent Behavior, 11, 151-166.

White, J. W. (2009). A gendered approach to adolescent dating violence: Conceptual and methodological issues. Psychology of Women Quarterly, 33, 1-15.

Wolfe, D. A., Crooks, C. C., Chiodo, D., \& Jaffe, P. (2009). Child maltreatment, bullying, gender-based harassment, and adolescent dating violence: Making the connections. Psychology of Women Quarterly, 33, 21-24.

Zurbriggen, E. L. (2009). Understanding and preventing adolescent dating violence: The importance of developmental, sociocultural, and gendered perspectives. Psychology of Women Quarterly, 33, $30-33$. 